\title{
Top-down modulation of the auditory steady-state response in a task-switch paradigm
}

\author{
Nadia Müller ${ }^{*}$, Winfried Schlee ${ }^{1}$, Thomas Hartmann', Isabel Lorenz' and Nathan Weisz ${ }^{2}$ \\ Department of Psychology, University of Konstanz, Konstanz, Germany \\ Zukunftskolleg, University of Konstanz, Konstanz, Germany
}

Edited by:

Olivier Bertrand, INSERM, France;

Lyon I University, France

Reviewed by:

Aurélie Bidet-Caulet, INSERM, France;

University of Lyon, France

Bernhard Ross, Rotman Research

Institute, Canada

\section{${ }^{*}$ Correspondence:}

Nadia Müller, Department of

Psychology, University of Konstanz,

Feursteinstr. 55 (ZPR, Haus 22), 78479

Konstanz, Germany.

e-mail: Nadia.Mueller@uni-konstanz.de
Auditory selective attention is an important mechanism for top-down selection of the vast amount of auditory information our perceptual system is exposed to. In the present study, the impact of attention on auditory steady-state responses is investigated. This issue is still a matter of debate and recent findings point to a complex pattern of attentional effects on the auditory steady state response (aSSR). The present study aimed at shedding light on the involvement of ipsilateral and contralateral activations to the attended sound taking into account hemispheric differences and a possible dependency on modulation frequency. In aid of this, a dichotic listening experiment was designed using amplitude-modulated tones that were presented to the left and right ear simultaneously. Participants had to detect target tones in a cued ear while their brain activity was assessed using MEG. Thereby, a modulation of the aSSR by attention could be revealed, interestingly restricted to the left hemisphere and $20 \mathrm{~Hz}$ responses: contralateral activations were enhanced while ipsilateral activations turned out to be reduced. Thus, our findings support and extend recent findings, showing that auditory attention can influence the aSSR, but only under specific circumstances and in a complex pattern regarding the different effects for ipsilateral and contralateral activations.

Keywords: aSSR, attention, MEG, suppression

\section{INTRODUCTION}

In daily life our brain is constantly exposed to a huge amount of sensory information which our perceptual system has to deal with. Due to limited processing capacities the sensory input has to be selected and structured, so that the brain becomes able to effectively cope with its environment. Selective attention is a key mechanism realizing these selection processes and has been intensively studied in the past Giard et al. (2000). Accordingly, neuronal activity of the relevant stimuli or stimuli features needs to be enhanced and processing facilitated whereas activity of distracting components ought to be suppressed. Indeed, attention to auditory stimuli was shown to induce an amplification of neuronal activity elicited by relevant stimuli (Petkov et al., 2004; Woldorff et al., 1993) and an inhibition of neuronal activity related to irrelevant stimuli (Bidet-Caulet et al., 2007) in auditory brain regions. Even though abundant evidence exists demonstrating the modulation of neuronal activity by attention in the auditory system, it is not clear at what level of the auditory system this happens. Empirical data from neuroimaging as well as electrophysiology has not been conclusive and only few studies were able to find involvement of auditory cortex (Bidet-Caulet et al., 2007; Fujiwara et al., 1998; Jäncke et al., 1999). Thus, for a better understanding of the mechanisms involved in auditory attention, it remains interesting if and how primary and secondary auditory cortices are contributing.

An elegant way of studying attentional effects in the auditory cortex is examining the auditory steady state response (aSSR). aSSRs are evoked by rapid periodic stimulus sequences in contrast to transient evoked responses. Usually, aSSRs are elicited by sequences of clicks (Galambos et al., 1981), amplitude modulated tones (Picton et al., 1987) or tone pulses (Pantev et al., 1996).

The generation of the aSSR was pinpointed to the auditory cortex. Studying this more precisely, it turned out that different parts of the auditory cortex are activated according to different modulation frequencies (Giraud et al., 2000). Liégeois-Chauvel et al. (2004) systematically studied the origin of steady-state responses generated by different AM frequencies: when looking at the left and right primary auditory cortex the amplitude of the steady-state response decreases continuously with increased modulation frequencies. The power of aSSRs modulated by $16 \mathrm{~Hz}$ or more are marginal in the right secondary auditory cortex. In the left secondary auditory cortex, however, the major power decline starts at $30 \mathrm{~Hz}$ - pointing to an impact of the left secondary auditory cortex in the generation of aSSRs with frequencies below $30 \mathrm{~Hz}$. These findings are in line with the intracranial studies conducted by Bidet-Caulet and colleagues who localized 21 and $29 \mathrm{~Hz}$ responses to primary and secondary areas of the auditory cortex. Furthermore, magnetic source imaging studies pinpoint the origin of the $40 \mathrm{~Hz}$ aSSR to the primary auditory cortex (Gutschalk et al., 1999; Pantev et al., 1996; Weisz et al., 2004; Wienbruch et al., 2006). Thus, steady-state responses of higher frequencies (gamma range) seem to be mainly generated in the primary auditory cortex whereas aSSRs of lower frequencies $(<30 \mathrm{~Hz})$ seem to have an origin in primary as well as secondary auditory cortex.

The analysis of the aSSR entails several advantages due to the characteristics of the resulting neuronal response: aSSRs closely follow the rhythm of the tone. Hence, knowing the modulation frequency, data analysis can be based on this predefined frequency. 
Since the noise is not phase-locked to the modulation frequency of the stimulus, averaging several responses leads to suppression of noise and thus to a strong signal-to-noise ratio. For MEG data in the auditory system it has been found that modulation frequencies around $40 \mathrm{~Hz}$ result in the strongest signal-to-noise ratio (Ross et al., 2000). According to the original work of Galambos et al. (1981), the steady-state response amplitude peaks between 15 and $20 \mathrm{~Hz}$ and again between 30 and $50 \mathrm{~Hz}$ with the major peak at $40 \mathrm{~Hz}$. Thus, the signal-to-noise ratio and the power of steady-state responses vary with the modulation frequency.

A further advantage of the aSSR is that multiple auditory stimuli with different modulation frequencies can be presented simultaneously leaving different traces in the recorded signal at the respective modulation frequencies. This technique termed 'frequency tagging' has been successfully employed in auditory neuroscience such as binaural integration (Fujiki et al., 2002), aversive conditioning (Weisz et al., 2007) or auditory stream segregation (Bidet-Caulet et al., 2007). This approach is especially interesting as in real life situations usually various auditory stimuli reach both ears at the same time, so that our brain has to focus on essential parts of the auditory information while ignoring distractor auditory stimuli. Using frequency-tagged stimuli, it is possible to simultaneously expose the auditory system to different tones and estimate the accordant power changes in dependence of the attentional load. Hence, for a better understanding of attentional processes in the primary and secondary auditory cortex, the investigation of if and how the aSSR is modulated by attention is essential.

Until now, little evidence exists in favour of an attentionmediated influence on the aSSR. In a pioneering EEG study, Linden et al. (1987) have not been able to disclose an attentional impact on the amplitude of the aSSR (stimulus rates $37-41 \mathrm{~Hz}$ ) despite a large variety of different employed paradigms. After this first authorative attempt, it took $>20$ years to show that it is indeed possible to modulate the $40 \mathrm{~Hz}$ aSSR by directed attention. In a MEG study, Ross et al. (2004) found an enhancement of the aSSRs amplitude by attention in the left hemisphere, contralateral to the auditory stimulation. Though the work of Ross and colleagues represents a significant step concerning the investigation of the attentional affect on the aSSR, their results are only informative to some extent. Thus, in their experimental setting, the aSSR may be affected by attentional changes that are not specific to the processed information but could result from more general changes in arousal or alertness. Moreover, selective attention could not be investigated within the auditory modality as the control task merely required attention to the visual domain. Finally, as they exclusively stimulated monaurally hemispheric differences could not be derivated.

Recently, Bidet-Caulet et al. (2007) did an illuminating study clarifying most of these open questions. Recording intracranial EEG in epilepsy patients they studied the mechanisms of selective attention in the primary auditory cortex. Their subjects were exposed to two competing auditory streams (stimulus rates 21 and $29 \mathrm{~Hz}$ ) and had to indicate the spatial direction of one of these two streams. The authors found an enhancement of the aSSR elicited by the attended stream and a reduction for the ignored stream. Interestingly, these results were restricted to the left hemisphere while the findings in the right hemisphere were more ambiguous. In line with this, accumulating evidence demonstrated that the left hemisphere appeared to be more sensitive to attentional modulation than the right hemisphere (Bidet-Caulet et al., 2007; Petkov et al., 2004). Furthermore, Skosnik et al. (2007) recently performed a study that investigated the impact of attention on 20 and $40 \mathrm{~Hz}$ responses. Click trains were presented binaurally in an oddball discrimination task and participants had to count targets $(20 \%$ of the stimuli). When the $40 \mathrm{~Hz}$ clicks were defined as targets the $40 \mathrm{~Hz}$ responses were enhanced while the amplitude of the $20 \mathrm{~Hz}$ responses did not change at frontocentral electrodes. In contrast, when participants were attending the $20 \mathrm{~Hz}$ responses, no significant power changes were observed for none of the responses.

Based on these recent results, it becomes clear that the aSSR is indeed modifiable by attention contrary to former assumptions of an insusceptibility of the aSSR to attention. Thereby, the modulation frequency, kind of task, experimental design or hemispheric differences turned out to be crucial for the attentional modulation of the aSSR. Nevertheless, various questions, clarifying the complex pattern of aSSRs and attention, are still open: the role of contralateral and ipsilateral activations contributing to the changes in the aSSR amplitude by attention is not solved yet. Furthermore, the susceptibility of the aSSR to attention is likely to change according to different modulation frequencies. This is interesting with regard to the varying impact of primary and secondary auditory cortex in the generation of steady-state responses elicited by different modulation frequencies.

In the present study, subjects were exposed to tones modulated by 20 and $45 \mathrm{~Hz}$ which were delivered to the right and left ear simultaneously. Subjects were asked to attend to a cued ear. In this way, both hemispheres were activated at the same time and changes in the aSSR amplitude could be derivated. These changes in amplitude were exclusively due to whether the respective AM tone was attended or not. Thus, our experimental design allowed for studying auditory selective attention within the auditory system in a situation of sound rivalry and to scrutinize on a possibly different behaviour of ipsilateral and contralateral activations. Furthermore, a possibly different sensitivity of the two hemispheres to attentional processes could be investigated. Finally, we were able to study attentional differences of the 20 and $45 \mathrm{~Hz}$ responses what is especially informative with respect to the different generators of these two steady-state responses.

\section{MATERIALS AND METHODS PARTICIPANTS}

Fifteen participants who reported normal hearing were included in the current study (nine male, six female). Mean age was 25 years (range 20-28 years). All participants were right handed according to the Edinburgh Handedness Inventory (Oldfield, 1971) and free of psychiatric or neurological disorders according to the M.I.N.I. (Mini International Neuropsychiatric Interview, German Version 5.0.0). Subjects were informed about the experimental procedure approved by the Ethical Committee of the University of Konstanz and signed a consent form before participating. After the experiment they were paid for participation. Two subjects had to be excluded because of too many artefacts in the MEG.

\section{STIMULI}

We exposed the participants to sinusoidal amplitude-modulated tones, modulated by 20 or $45 \mathrm{~Hz}$, respectively. The carrier frequency 
of both stimuli was set to $655 \mathrm{~Hz}$. Stimulus duration was $800 \mathrm{~ms}$. Tones were presented with a sampling rate of $44,100 \mathrm{~Hz}$ and a modulation depth of $100 \%$. To avoid clicks at the on- and offset of the stimuli we included a $50 \mathrm{~ms}$ fade-in period at the beginning of the tone and a $50 \mathrm{~ms}$ fade-out period at the end of the tone. Participants listened to tones on both ears simultaneously in a way that the $20 \mathrm{~Hz}$ modulated tone was presented to one ear and the $45 \mathrm{~Hz}$ modulated one to the other ear. The side of stimulation was alternated randomly and equally balanced between tones and ears. As a result, in half of the trials, the $20 \mathrm{~Hz}$ modulated tone was presented to the left while the $45 \mathrm{~Hz}$ modulated one was given to the right ear. Accordingly, for the other half of the trials the configuration of the stimuli was reversed. In $10 \%$ of the cases one of the tones was replaced by a target. A target was defined as a tone that altered its modulation frequency during stimulation. To clarify, the tone's amplitude was modulated by either 45 or $20 \mathrm{~Hz}$, changed after $0.2 \mathrm{~s}$ to 25 or $12.5 \mathrm{~Hz}$ respectively and returned to its initial modulation rate after $0.6 \mathrm{~s}$.

To provide the same intensity to each ear and each person, tones were adjusted to the individual hearing level in both ears separately. Intensity was chosen $50 \mathrm{~dB}$ above sensation level.

\section{EXPERIMENTAL DESIGN AND PROCEDURE}

The experimental configuration resulted in a $2 \times 2$ design: two attentional conditions (attend tone vs. ignore tone) and two conditions arising from the different modulation frequencies $(20 \mathrm{~Hz}$ vs. $45 \mathrm{~Hz}$ ) of the steady-state tones.

For attentional modulation a task-switch paradigm was used. Subjects saw an arrow pointing randomly to the left or to the right that told them to shift their attention to the designated ear. Subjects' task was to detect target tones (10\%) that were defined as tones altering their modulation frequency in between presentation in the attended ear. Trials with target tones were later excluded from statistical analysis.

The different conditions were equally weighted and a total of 200 trials presented during the course of the experiment. The presentation order of the trials was randomized. Each trial commenced with a cross in the middle of the screen that subjects had to fixate for 1-1.5 s. Subsequently, an arrow, pointing to the right or left side, was shown to indicate the focus of attention. After $1-1.5 \mathrm{~s}$ subjects were exposed to the auditory stimulus. Directly afterwards, subjects were asked if they could detect a target or not by displaying the question in the screen. Thereupon subjects had to respond with a right-hand button press; left key meant 'target' and right key 'no target'. Consequently, if the tone in the designated ear altered its modulation frequency in between presentation subjects had to respond by pressing the left button, otherwise by the right button. Subjects were not forced to respond as fast as possible. The intertrial interval (ITI) varied between 2.5 and 3.5 s. During the ITI, subjects saw a pair of eyes that encouraged them to blink so that blinking was avoided during task performance. The time intervals, which the fixation cross, the arrow or the pair of eyes appeared in, differed slightly in a randomised manner to prevent expectation effects. The procedure of one trial is illustrated in Figure 1.

\section{DATA ACOUISITION}

Stimulus presentation and presentation of the instructions was programmed using Psyscope X, Version Dep. 37 (Cohen et al., 1993), an open-source environment for the design and control of behavioural experiments (http://psy.ck.sissa.it/). Triggers were generated in Psyscope and sent to the data acquisition system via the trigger box of the MEG. Tones were generated outside of the magnetic shielded chamber (ASG-BTI) and delivered to the subject's ear via flexible plastic tubes of the sound system. Instructions and visual stimuli were presented by a video beamer (JVCTM, DLA-G11E) outside of the MEG chamber and projected to the ceiling in the MEG chamber by means of a mirror system. For their responses subjects used a response pad that recorded the responses separately. Individual head shapes were collected from all subjects using a digitizer. The MEG recordings were accomplished with a 148-channel magnetometer system (MAGNESTM $2500 \mathrm{WH}, 4 \mathrm{D}$ Neuroimaging, San Diego, CA, USA), installed in a magnetically shielded chamber (Vakuumschmelze Hanau), while participants lay in a supine position. MEG signals were recorded with a sampling rate of $678.17 \mathrm{~Hz}$ and a hard-wired high-pass filter of $0.1 \mathrm{~Hz}$.

\section{DATA ANALYSIS}

Data sets were exported to Matlab (The MathWorks, Natick, MA, USA, Version 7.5.0 R 2007b) for processing. Epochs of $2 \mathrm{~s}$ pre and $2 \mathrm{~s}$ post-stimulus were cut out of the continuous data streams. This was done for different conditions separately, so that there were 45 trials for each of the four conditions. Target trials were

\section{Procedure of one trial}

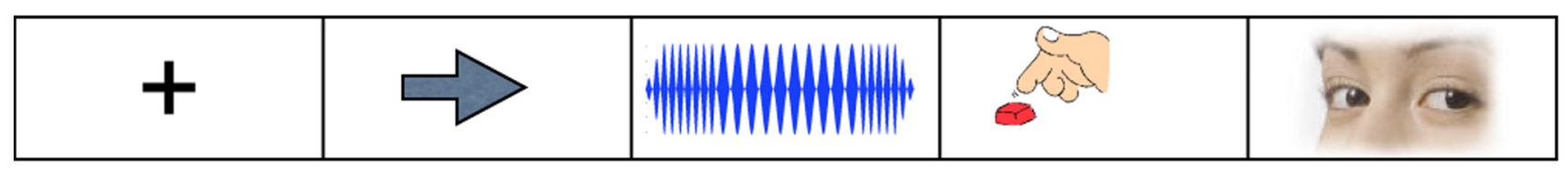

pre-cue period pre-stimulus period tone stimulation intertrial interval
$1000-1500 \mathrm{~ms}$
$1000-1500 \mathrm{~ms}$
$800 \mathrm{~ms}$
$1500 \mathrm{~ms}$
$2500-3500 \mathrm{~ms}$

FIGURE 1 | Illustration of a target trial used in the experiment. 
excluded from statistical analysis. The resulting trials were visually inspected for artefacts and bad ones rejected. As subjects were asked to restrain blinking to the ITI and not to move during the experiment, the trials that had to be rejected were very limited. The signal of the 148 channels of the MEG was projected onto a montage of eight regional sources according to Weisz et al. (2007); for a general description of the concept of source montages see Scherg et al. (2002). In their approach a source model is generated consisting of eight sources, including the two sources of interest (left temporal: -0.680 .000 .06 and right temporal: 0.680 .000 .06 , coordinates in unit spheres) and six sources distributed over the remaining cortex. The sources outside of the regions of interest acted as a kind of spatial filter reducing the influence of non-auditory sources on the sources of interest. Thus, for further analysis of the aSSRs only the locations of interest (left and right temporal sources) were regarded. The location of the temporal sources was guided by previous localization works of the aSSR by our group (Weisz et al., 2007). After source projection data sets (containing three orthogonal dipoles) were oriented by performing a principal component analysis. For further analysis only the first orientation was taken into account.

Prior to the calculation of the aSSR fast Fourier transformation (FFT) was applied to the average of all unfiltered trials (i.e. the event-related field, ERF) for each condition and source. As time window an interval from 300 to $700 \mathrm{~ms}$ after tone onset was chosen, corresponding to the maximal steady-state activation revealed by an exploratively performed wavelet analysis (Morlet wavelet with an $\mathrm{m}$-factor of 7; this factor regulates the 'compromise' between time and frequency resolution) applied to the ERF (Bertrand and Tallon-Baudry, 2000). Both Fast Fourier Transformed data and Wavelet Transformed data were baseline corrected. As baseline served an interval prior to cue presentation (from 700 to $300 \mathrm{~ms}$ before cue onset) while subjects were regarding a fixation cross. Wavelet transformed data was baseline corrected by dividing the power values during tone stimulation by the power values of the baseline interval. Fourier Transformed data was baseline corrected by subtracting the power values of the baseline interval from the power values during tone stimulation.

For statistical analysis, a $2 \times 2$ ANOVA was performed using a nonlinear mixed effects model (Pinheiro and Bates, 2000). The factors 'attention', 'hemisphere', 'laterality' and 'modulation frequency' were defined as fix factors, whereas the factor 'subject' represented the random factor. Thereby, the amplitudes of the steady-state responses in dependence of the hemisphere (left vs. right), laterality (ipsilateral vs. contralateral), modulation frequency ( $20 \mathrm{~Hz}$ vs. $45 \mathrm{~Hz}$ ) and attention (attend vs. ignore) were analysed. Attention was defined as attention to a specific modulation frequency. Additionally, paired Student's $t$-tests were performed for a post hoc analysis of the effects. Significance level was set at $5 \%$.

\section{RESULTS}

\section{BEHAVIOURAL RESULTS}

The experimental task was challenging for the participants as can be demonstrated by the behavioural results. Over all subjects and trials $74 \%$ of the tones were identified correctly. Thus, subjects were able but had to make an effort to distinguish targets from standard tones. Subjects showed the same behavioural performance for the fast (mean \pm standard deviation $76 \pm 18 \%$ ) and the slow tones $(72 \pm 23 \%)$. Likewise, attending to the left $(73 \pm 20 \%)$ or to the right ear $(76 \pm 18 \%)$ did not affect the respective response patterns. The equivalence was confirmed statistically by the appropriate Student's $t$-tests. Both tests argue for the absence of differences between means (each $p>0.5$ ).

\section{ELECTROPHYSIOLOGICAL DATA}

An explorative time-frequency analysis of the ERFs showed prominent power increases at the modulation frequencies. The 20 and $45 \mathrm{~Hz}$ responses peaked around 300 and $700 \mathrm{~ms}$ post-stimulus. An illustration of the power distribution of the mean steady-state response over all subjects and conditions is shown in Figure 2.

As the outcome of time-frequency analyses always is a compromise between time vs. frequency resolution a smearing of nearby frequencies could not be ruled out, e.g. between the $45 \mathrm{~Hz}$ response and the harmonics of the $20 \mathrm{~Hz}$ responses at $40 \mathrm{~Hz}$. Therefore, the time window (300-700 ms post-stimulus) of relevant energy increase identified by this step was used and FFTs producing power estimates for discrete frequencies were applied to our data sets (with the cost of losing time information). Thereby, we could infer frequency-specific activations more precisely: neuronal activity was significantly enhanced at the frequencies of modulation and its first harmonics. Thus, besides the 20 and $45 \mathrm{~Hz}$ responses, neuronal activity was augmented at $40 \mathrm{~Hz}$, the first harmonic of the $20 \mathrm{~Hz}$ response. For an illustration see Figure 3.

For a detailed investigation of the attentional effects on the aSSR the possibly different effect of ipsilateral and contralateral activations, 20 and $45 \mathrm{~Hz}$ responses as well as left hemispheric and right hemispheric activations were taken into account by calculating a nonlinear mixed effects model including these factors. Thereby we revealed two main effects: contralateral activations were significantly larger than ipsilateral activations $(F=9.10$, $p=0.003)$ and left hemispheric activations significant larger than right hemispheric activations $(F=5.02, p=0.03)$. There were no main effects for modulation frequency and attention. To find out if the significant main effects are due to attentional processes we looked at the accordant interactions: there was a first order interaction for laterality $\times$ attention $(F=4.30, p=0.04)$, showing that aSSRs contralaterally to tone presentation are enhanced by attention and aSSRs ipsilaterally to tone presentation reduced by attention. When looking closer at higher order interactions (later ality $\times$ attention $\times$ hemisphere: $F=5.18, p=0.02$ ) it becomes clear that attention seems to act differently depending on the interplay between ipsilateral and contralateral as well as right temporal and left temporal activations. Therefore, we assessed differences in the aSSR amplitude due to attention for left hemispheric and right hemispheric as well as at 20 and $45 \mathrm{~Hz}$ responses separately by calculating the accordant Student's $t$-tests. Thereby, a hemispheric and frequency-specific impact of attention on the auditory steadystate response could be disclosed. First, the power of the $20 \mathrm{~Hz}$ responses was not affected by attention in the right hemisphere. In contrast, looking at the left hemisphere, the $20 \mathrm{~Hz}$ response was shown to be significantly modulated by attention: regarding $20 \mathrm{~Hz}$ responses contralateral to tone presentation in the right ear we could demonstrate a significant enhancement of the aSSR caused by attention. That is, attending to a specific tone in the right ear 


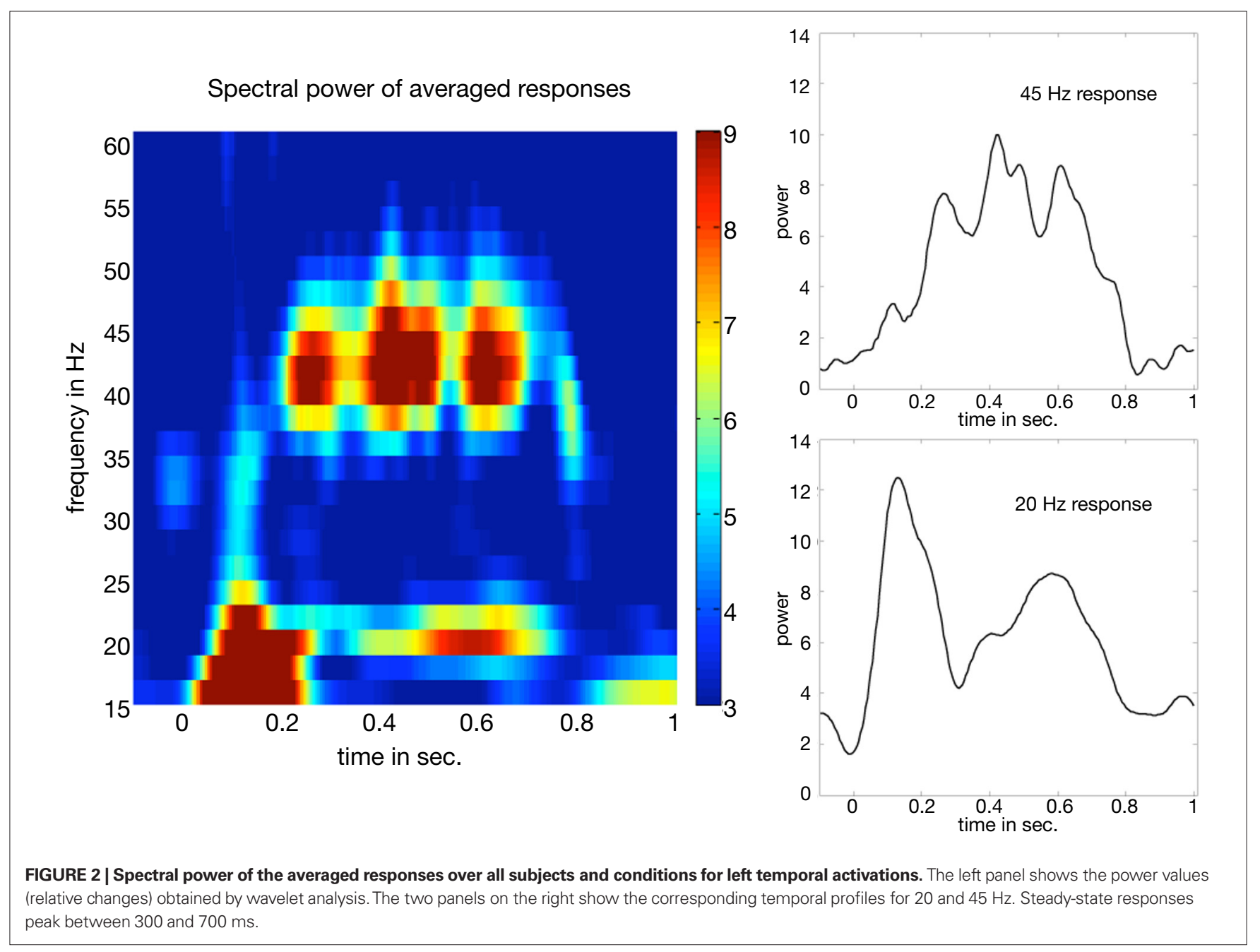

led to an enhancement of the amplitude of the $20 \mathrm{~Hz}$ steady-state responses in the left hemisphere. In contrast, if the attended sound was presented to the left ear the $20 \mathrm{~Hz}$ activations appeared to be significantly reduced by attention in the left hemisphere. The suppression-effect was even more pronounced than the enhancement effect. See Figure 4 for an illustration.

Looking at the $45 \mathrm{~Hz}$ responses it seems at a first glance that the aSSRs are enhanced when attending to the contralateral right ear. However, the accordant Student's $t$-test could not reveal any power changes due to attention (all $p>0.1$ ). This was the case for both hemispheres. See Figure 5 for an illustration.

\section{DISCUSSION}

With the present work we demonstrate that top-down modulated auditory spatial selective attention affects the power of the aSSR in a specific pattern: attending to the right ear resulted in a contralateral enhancement of the $20 \mathrm{~Hz}$ aSSR in the left hemisphere while attending to the left ear led to an ipsilateral reduction of the $20 \mathrm{~Hz}$ aSSR in the left hemisphere. The right hemisphere was unaffected by attention. Furthermore, no significant effects were found for $45 \mathrm{~Hz}$ neither in the left nor in the right hemisphere. This complex pattern supports previous studies and might help to explain some of the inconsistencies in the existing literature.

\section{ATTENTIONAL MODULATION OF THE aSSR}

The present study addresses several aspects concerning attentional processes in the auditory cortex and thereby adds to previous findings. As has been illustrated in the introduction part some recent studies (Bidet-Caulet et al., 2007; Ross et al., 2004; Skosnik et al., 2007) indicate that it is indeed possible to modulate the aSSR by attention. This was not obvious as after negative results in a very early study (Linden et al., 1987) the aSSR was thought to be insusceptible to attention.

This insusceptibility, however, was later substantiated in the experimental task used by Linden and colleagues: their subjects solely concentrated on changes in carrier frequency. In contrast, forcing subjects to attend to the stimulus rhythm was an important factor in the modulation of the aSSR. Thus, by using an appropriate task, that means a task that makes subjects attending to the modulation frequency of the stimulus, it was possible to show a susceptibility of the aSSR to attention. 


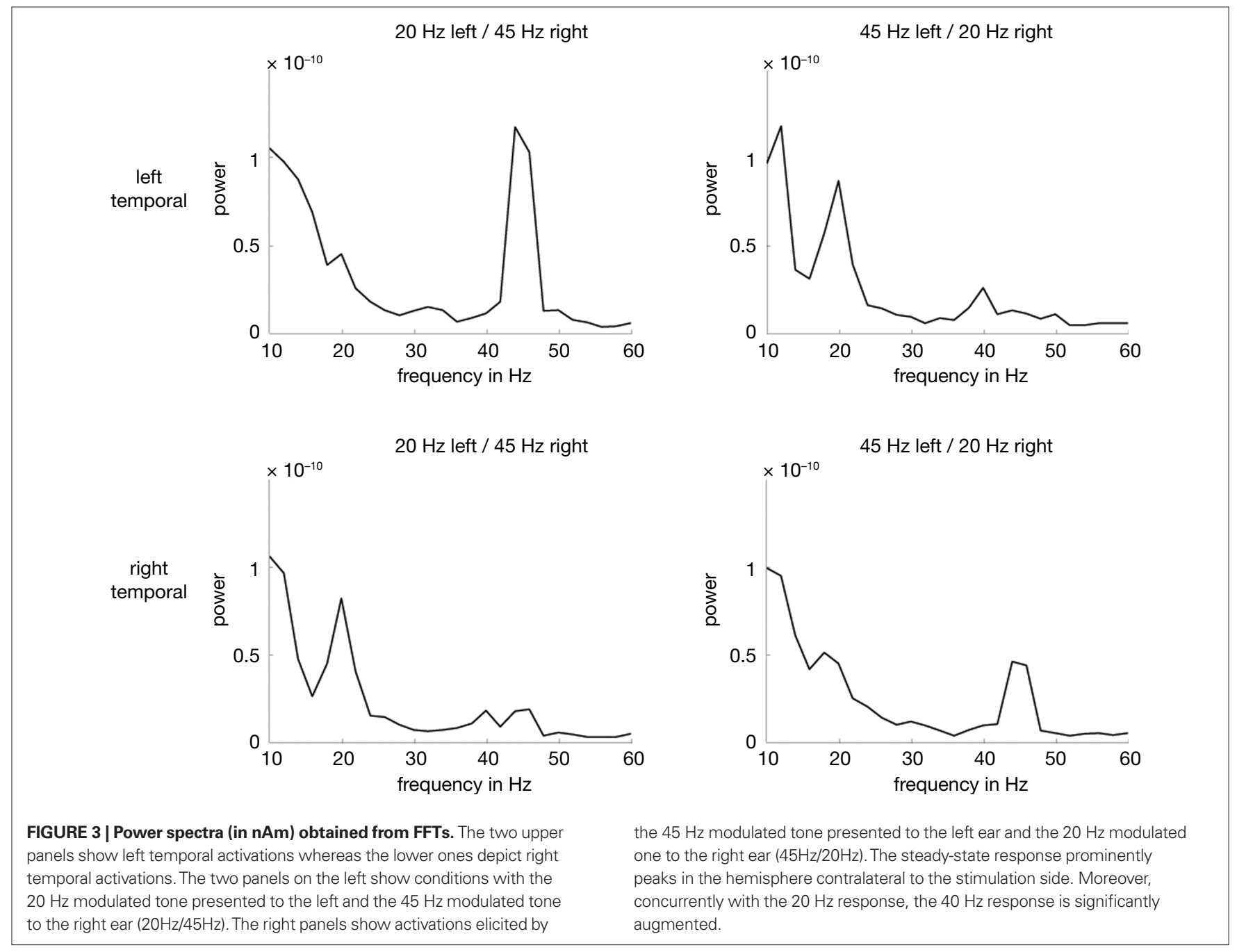

\section{$20 \mathrm{~Hz}$ responses}

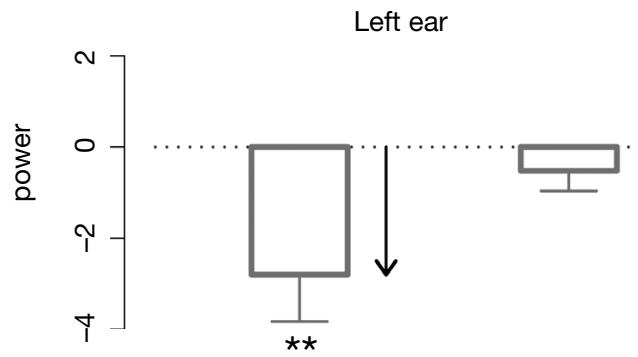

left temporal right temporal
FIGURE 4 | Differences in mean power values between the attended and ignored $20 \mathrm{~Hz}$ aSSR (attend-ignore). Activations in the left temporal cortex are significantly modulated by attention: following a right cue the steady-state

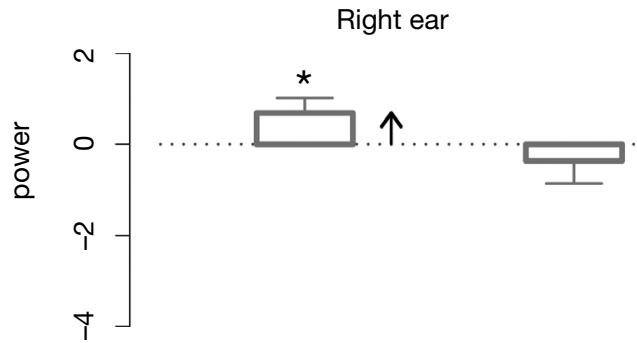

left temporal right temporal
But still, the pattern of significant findings appears to be too complex to be explained by some unspecific attentional influences. Attentional modulation is highly dependent on experimental factors, amplitude is enhanced in the left hemisphere. For a left cue, however, the left temporal $20 \mathrm{~Hz}$ responses are reduced. In the right hemisphere no differences due to attention could be revealed. 
$45 \mathrm{~Hz}$ responses

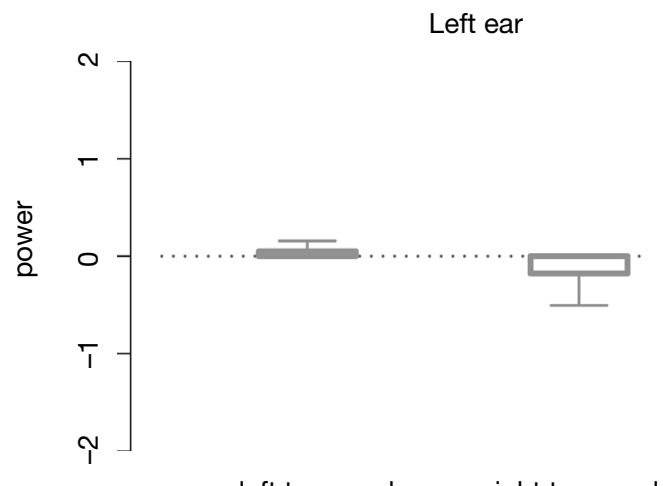

left temporal

right temporal

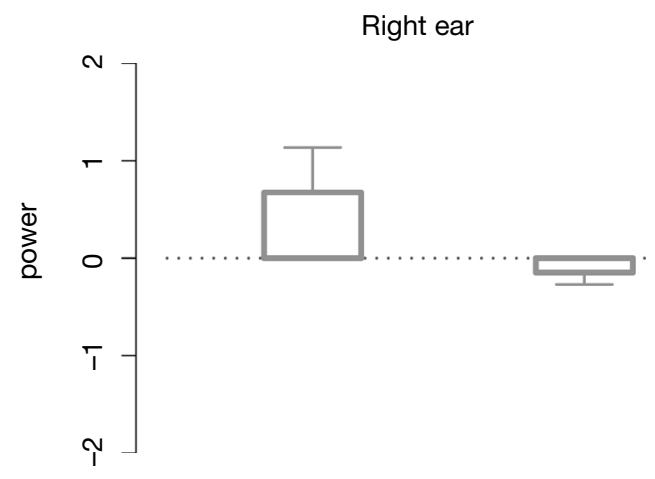

left temporal right temporal

FIGURE 5 | Differences in mean power values between the attended and ignored $\mathbf{4 5} \mathbf{~ H z}$ aSSR (attend-ignore). No statistical significant differences due to attention were found neither for the left nor for the right hemisphere.

discussion it therefore seems to be essential to scrutinize on the factors that influence the attentional impact on the aSSR. Our experimental design allowed for investigating top-down modulation of the aSSR with respect to different modulation frequencies and to ipsilateral and contralateral activations within the auditory modality.

\section{ENHANCEMENT VERSUS SUPPRESSION OF THE aSSR}

Until now, only few studies exist investigating the relationship between attention and the aSSR. To our knowledge, none of these studies have reported effects on ipsilateral and contralateral activations separately. By our experimental design, however, it is possible to dissolve the apparently complex activation patterns during top-down processes and scrutinize on ipsilateral and contralateral as well as left hemispheric and right hemispheric activations. The $20 \mathrm{~Hz}$ responses are significantly enhanced in the left hemisphere when subjects had to attend to the right ear. This is in line with the findings of Ross et al. (2004) who showed a left hemispheric enhancement of the aSSR following contralateral right ear stimulation. It furthermore supports the results of Bidet-Caulet et al. (2007), Skosnik et al. (2007) and Weisz, submitted who all report an amplitude augmentation of the attended aSSRs.

Additionally to this enhancement effect, the $20 \mathrm{~Hz}$ responses were significantly suppressed in the left hemisphere when subjects had to attend to the ipsilateral left ear. As depicted above there is no study investigating selective attention in the auditory domain with respect to ipsilateral and contralateral activations. In the somatosensory modality, however, Staines et al. (2002) revealed note-worthy results concerning ipsilateral inhibition of relevant information. The experimental design used by Staines and colleagues was similar to the one used in the present experiment. Subjects were exposed to vibrating little hammers at a frequency of $25 \mathrm{~Hz}$ and had to detect targets defined as brief changes in frequency. The authors could reveal two main effects: a facilitation of activity in the primary somatosensory cortex contralateral to the stimulated hand and an inhibition ipsilateral to the stimulation side. This is in line with our findings of ipsilateral suppression and contralateral enhancement. Apart from this study little is known about suppression of
SSRs when attending to ipsilaterally presented stimuli. However, the principle of enhancing relevant and suppressing irrelevant neuronal activity for a better signal-to-noise ratio is not new and represents a key mechanism for effective information processing. This issue is further addressed in the next section.

\section{COMPETITION OF NEURONAL RESOURCES AND SUPPRESSION OF ACTIVITY}

Competing for neuronal resources seems to be a basic principle in the organisation of neuronal systems. Competition is found on the single cell level of neurons' receptive fields (Reynolds et al., 1999), on the intrahemispheric level within modalities (Kastner et al., 1998), between the two hemispheres (Allison et al., 2000; Brancucci et al., 2004) and between different modalities (Ghatan et al., 1998). Thus, it is not surprising that if the aSSR is affected by attention, this top-down modulation not simply results in an enhancement of the steady-state response, but also involves suppression processes. Enhancing relevant and suppressing irrelevant input in the processing of aSSRs is in line with the recent findings of Bidet-Caulet et al. (2007) who found an augmentation of the relevant and a reduction of the irrelevant aSSR in the left hemisphere during a situation of sound rivalry. In contrast to Bidet-Caulet's study our experimental task required attention to a given ear instead of attention to a given stimulus. Thus, it is plausible that in this case neuronal activity in the contralateral hemisphere where main processing takes place is highlighted while possibly interfering activity in the ipsilateral hemisphere is suppressed. In situations of high competition as sound rivalry (Bidet-Caulet et al., 2007) or task-switching (Rykhlevskaia et al., 2006) the suppression of interfering activity is at least as crucial as the enhancement of relevant information for effective information processing. This is reflected by our data showing an even stronger suppression than enhancement effect.

\section{LEFT HEMISPHERIC DOMINANCE IN ATTENTIONAL PROCESSES}

The $20 \mathrm{~Hz}$ responses were significantly modulated by attention, but this was only the case for the left hemisphere. In the right hemisphere no significant differences due to attention could be revealed. 
This appears surprising at a first glance, but corroborates previous reports in the literature: all existing studies looking at aSSRs and attention report a more pronounced and consistent modulation in the left hemisphere than the right hemisphere (Bidet-Caulet et al., 2007; Ross et al., 2004). Bidet-Caulet and colleagues even found paradoxical effects pointing to a reversed pattern of steady-state modulation in the right hemisphere. Moreover, a left hemispheric dominance in top-down selection processes in the auditory system was found in various studies (Coch et al., 2005; Petkov et al., 2004). Petkov et al. (2004) conducted a fMRI study using high-resolution surface mapping techniques and have shown that attended stimuli elicited larger enhancements of neuronal responses in the left hemisphere whereas unattended stimuli displayed greater activations in the right hemisphere. Attentional modulations were specific to non-primary auditory regions. In a study of Weisz, submitted enhancements of aSSRs due to attention were also particularly observed for the left hemisphere. Thus, in the auditory modality, the left hemisphere seems to be more susceptible to attentional selection processes than the right hemisphere what is confirmed by our principle findings.

\section{MODULATION-FREQUENCY DEPENDENCY OF ATTENTIONAL EFFECTS}

As stated above the $20 \mathrm{~Hz}$ response was modulated by attention in a way expected from previous studies (Bidet-Caulet et al., 2007). The $45 \mathrm{~Hz}$ response, however, was not modulated by our experimental design. This does not contradict previous positive findings with modulation frequencies around $40 \mathrm{~Hz}$ of Ross et al. (2004), Skosnik et al. (2007) and Weisz, submitted as our experimental design differed in various aspects.

By presenting an informative vs. an uninformative cue Weisz and colleagues manipulated the attentional load prior to tone presentation, but subjects had to detect and thus attend to the $42 \mathrm{~Hz}$ tone in both conditions. Consequently they never really ignored the $42 \mathrm{~Hz}$ tone as subjects had to in our experiment. In the present study attention switched between ears and all cues were predictive of target location, i.e. no comparison can be made between informative and uninformative cueing as in the Weisz et al. study. These differences in design obviously can lead to different results. Skosnik and colleagues binaurally presented click trains that were modulated by either 20 or $40 \mathrm{~Hz}$ and found attentional effects exclusively for the $40 \mathrm{~Hz}$ responses. This was in contrast to our results that showed the $20 \mathrm{~Hz}$ responses being modulated by attention. However, as they did not present the 20 and $40 \mathrm{~Hz}$ click trains simultaneously, they did not elicit a situation of sound rivalry as it was induced in the present design. Moreover, as the authors selected an oddball paradigm to modulate attention, attended tones (targets) were at the same time novel. Thus their effects could also be caused by bottom-up processes as Skosnik and colleagues already stated. The described differences to our study could explain the different results. The findings of Ross and colleagues cannot be paralleled to our experimental design either as they used a visual control task which did not allow for investigating attention within the auditory modality. Thus, all these findings do not contradict the present results.

The only experimental design that could be paralleled to ours is the investigation of Bidet-Caulet et al. (2007). However, they found attention-mediated effects for the 21 and $29 \mathrm{~Hz}$ responses and did not look at higher frequencies.
Thus, the question why $20 \mathrm{~Hz}$ responses are differently modulated by attention than $45 \mathrm{~Hz}$ responses using the present experimental design remains a matter of further investigation: at this point some speculations will be made in an attempt to resolve this issue: first, different parts within the auditory system contribute to the generation of the $20 \mathrm{~Hz}$ response and the $45 \mathrm{~Hz}$ response (Giraud et al., 2000). According to Liégeois-Chauvel et al. (2004) $45 \mathrm{~Hz}$ responses are generated mainly in the left and right primary auditory cortex. The $20 \mathrm{~Hz}$ responses, however, are generated in the left and right primary and left secondary auditory cortex. Moreover, intracranial data (Bidet-Caulet et al., 2007) demonstrated that the $40 \mathrm{~Hz}$ response is exclusively found in the medial part of Hesch'l Gyrus while the $20 \mathrm{~Hz}$ response stems from medial and lateral parts of the Hesch'l Gyrus.

The studies of Liégeois-Chauvel et al. (2004) and Bidet-Caulet et al. (2007) definitely support a different generation of aSSRs around $40 \mathrm{~Hz}$ compared to the $20 \mathrm{~Hz}$ response. This could imply a varying sensitivity of different aSSRs to attentional modulation what can be translated into a different sensitivity of various auditory fields (generating the aSSR) to attentional modulation similar to what has been reported by Petkov et al. (2004) in an fMRI study. As only the left secondary auditory cortex has been shown to be a generator of the $20 \mathrm{~Hz}$ aSSR and as we exclusively found the $20 \mathrm{~Hz}$ response to be modulated by attention in the left auditory cortex, this could hint a susceptibility of the secondary auditory cortex to attention and at the same time an insusceptibility of the primary auditory cortex to attention.

If aSSRs with varying modulation frequencies are differently modifiable by attention due to the respective generators has to be confirmed systematically, but - if confirmed - would open up interesting opportunities for cognitive neuroscience to probe various auditory fields using the excellent temporal resolution of MEG/EEG.

\section{CONCLUSION}

Our results demonstrate that top-down modulated auditory spatial selective attention affects the power of the aSSR in a specific pattern: attending to the right ear resulted in a contralateral enhancement of the $20 \mathrm{~Hz}$ aSSR in the left hemisphere while attending to the left ear led to an ipsilateral reduction of the $20 \mathrm{~Hz}$ aSSR in the left hemisphere. For the right hemisphere no effects could be revealed supporting the left hemispheric specialization in auditory attention. Furthermore, $45 \mathrm{~Hz}$ responses seemed to be invariant to attention pointing to a modulation-frequency dependency in attentional modulation of the aSSR.

Hence, the present study could elucidate the interplay of contralateral and ipsilateral steady-state activations in auditory attention and thereby account for some of the inconsistencies in the present research of aSSRs and attention.

\section{ACKNOWLEDGEMENTS}

This research was supported by the Deutsche Forschungsgemeinschaft (DFG) and the Tinnitus Research Initiative (TRI). The authors would also like to thank Christina Robert for proofreading this manuscript.

\section{SUPPLEMENTARY MATERIAL}

The Supplemental Data for this article can be found online at http://www.frontiersin.org/humanneuroscience/paper/10.3389/ neuro.09.001.2009. 


\section{REFERENCES}

Allison, J. D., Meador, K. J., Loring, D. W., Figueroa, R. E., and Wright, J. C. (2000). Functional MRI cerebral activation and deactivation during finger movement. Neurology 54, 135-142.

Bertrand, O., and Tallon-Baudry, C. (2000). Oscillatory gamma activity in humans: a possible role for object representation. Int. J. Psychophysiol. 38, 211-223.

Bidet-Caulet, A., Fischer, C., Besle, J., Aguera, P., Giard, M., and Bertrand, O. (2007). Effects of selective attention on the electrophysiological representation of concurrent sounds in the human auditory cortex. J. Neurosci. 27, 9252-9261.

Brancucci, A., Babiloni, C., Babiloni, F., Galderisi, S., Mucci, A., Tecchio, F., Zappasodi, F., Pizzella, V., Romani, G. L., and Rossini, P. M. (2004). Inhibition of auditory cortical responses to ipsilateral stimuli during dichotic listening: evidence from magnetoencephalography. Eur. J. Neurosci. 19, 2329-2336.

Coch, D., Sanders, L. D., and Neville, H. J. (2005). An event-related potential study of selective auditory attention in children and adults. J. Cogn. Neurosci. 17, 605-622

Cohen, J., MacWhinney, B., Flatt, M., and Provost, J. (1993). PsyScope: an interactive graphical system for designing and controlling experiments in the psychology laboratory using macintosh computers. Behav. Res. Methods Instrum. Comput. 25, 257-271.

Fujiki, N., Jousmaki, V., and Hari, R. (2002). Neuromagnetic responses to frequency-tagged sounds: a new method to follow inputs from each ear to the human auditory cortex during binaural hearing. J. Neurosci. 22, RC205.

Fujiwara, N., Nagamine, T., Imai, M., Tanaka, T., and Shibasaki, H. (1998). Role of the primary auditory cortex in auditory selective attention studied by whole-head neuromagnetometer. Brain. Res. Cogn. Brain. Res. 7, 99-109.

Galambos, R., Makeig, S., and Talmachoff, P. J. (1981). A 40-Hz auditory potential recorded from the human scalp. Proc. Natl. Acad. Sci. U.S.A. 78, 2643-2647.

Ghatan,P.H.,Hsieh,J.C., Petersson, K. M. Stone-Elander, S., and Ingvar, M. (1998). Coexistence of attentionbased facilitation and inhibition in the human cortex. Neuroimage 7, 23-29.

Giard, M. H., Fort, A., MouchetantRostaing, Y., and Pernier, J. (2000). Neurophysiological mechanisms of auditory selective attention in humans. Front. Biosci. 5, D84-D94.

Giraud, A. L., Lorenzi, C., Ashburner, J. Wable, J., Johnsrude, I., Frackowiak, R. and Kleinschmidt, A. (2000). Representation of the temporal envelope of sounds in the human brain. J. Neurophysiol. 84, 1588-1598.

Gutschalk, A., Mase, R., Roth, R., Ille, N., Rupp, A., Hähnel, S., Picton, T.W., and Scherg, M. (1999). Deconvolution of $40 \mathrm{~Hz}$ steady-state fields reveals two overlapping source activities of the human auditory cortex. Clin. Neurophysiol. 110, 856-868.

Jäncke, L., Mirzazade, S., and Shah, N. J. (1999). Attention modulates activity in the primary and the secondary auditory cortex: a functional magnetic resonance imaging study in human subjects. Neurosci. Lett. 266, 125-128.

Kastner, S., De Weerd, P., Desimone, R., and Ungerleider, L. G. (1998). Mechanisms of directed attention in the human extrastriate cortex as revealed by functional MRI. Science $282,108-111$.

Liégeois-Chauvel, C., Lorenzi, C., Trébuchon, A., Régis, J., and Chauvel, P. (2004). Temporal envelope processing in the human left and right auditory cortices. Cereb. Cortex 14, 731-740.

Linden, R. D., Picton, T. W., Hamel, G. and Campbell, K. B. (1987). Human auditory steady-state evoked potentials during selective attention. Electroencephalogr. Clin. Neurophysiol. 66, 145-159.

Oldfield, R. C. (1971). The assessment and analysis of handedness: the Edinburgh inventory. Neuropsychologia 9, 97-113.

Pantev, C., Roberts, L.E., Elbert, T., Ross, B., and Wienbruch, C. (1996). Tonotopic organization of the sources of human auditory steady-state responses. Hear. Res. 101, 62-74.

Petkov, C. I., Kang, X., Alho, K., Bertrand, O., Yund, E. W., and Woods, D. L. (2004). Attentional modulation of human auditory cortex. Nat. Neurosci. 7, 658-663.

Picton, T. W., Skinner, C. R. Champagne, S. C., Kellett, A. J., and Maiste, A.C. (1987). Potentials evoked by the sinusoidal modulation of the amplitude or frequency of a tone. J. Acoust. Soc. Am. 82, 165-178.

Pinheiro, J., and Bates, D. M. (2000) Mixed-effects models in S and S-Plus. Springer. New York.

Reynolds, J. H., Chelazzi, L., and Desimone, R. (1999). Competitive mechanisms subserve attention in macaque areas V2 and V4. J. Neurosci. 19, 1736-1753.

Ross, B., Borgmann, C., Draganova, R. Roberts, L. E., and Pantev, C. (2000). A high-precision magnetoencephalographic study of human auditory steady-state responses to amplitudemodulated tones. J. Acoust. Soc. Am 108, 679-691.

Ross, B., Picton, T. W., Herdman, A. T., and Pantev, C. (2004). The effect of attention on the auditory steady-state response. Neurol. Clin. Neurophysiol. 2004, 22.

Rykhlevskaia, E., Fabiani, M., and Gratton, G. (2006). Lagged covariance structure models for studying functional connectivity in the brain. Neuroimage 30, 1203-1218.

Scherg, M., Ille, N., Bornfleth, H., and Berg, P. (2002). Advanced tools for digital EEG review: virtual source montages, whole-head mapping correlation, and phase analysis. J. Clin. Neurophysiol. 19, 91-112.

Skosnik, P. D., Krishnan, G. P., and O'Donnell, B. F. (2007). The effect of selective attention on the gammaband auditory steady-state response. Neurosci. Lett. 420, 223-228.

Staines, W. R., Graham, S. J., Black, S. E. and McIlroy, W. E. (2002). Task-relevant modulation of contralateral and ipsilateral primary somatosensory cortex and the role of a prefrontal-cortical sensory gating system. Neuroimage 15 190-199.
Weisz, N., Keil, A., Wienbruch, C., Hoffmeister, S., and Elbert, T. (2004). One set of sounds, two tonotopic maps: exploring auditory cortex with amplitude-modulated tones. Clin. Neurophysiol. 115, 1249-1258.

Weisz, N., Kostadinov, B., Dohrmann, K. Hartmann, T., and Schlee, W. (2007). Tracking short-term auditory cortical plasticity during classical conditioning using frequency-tagged stimuli. Cereb. Cortex 17, 1867-1876.

Weisz, N., Lecaignard, F., Müller N., and Bertrand O. (submitted). Primary auditory cortical brain activity is lateralized according to informational content of a spatial cue.

Wienbruch, C., Paul, I., Weisz, N., Elbert, T., and Roberts, L. E. (2006) Frequency organization of the 40$\mathrm{Hz}$ auditory steady-state response in normal hearing and in tinnitus. Neuroimage 33, 180-194.

Woldorff, M. G., Gallen, C. C., Hampson, S. A., Hillyard, S. A., Pantev, C., Sobel, D., and Bloom, F. E. (1993). Modulation of early sensory processing in human auditory cortex during auditory selective attention.Proc. Natl. Acad. Sci. U.S.A. 90, 8722-8726.

Conflict of Interest Statement: The authors declare that the research was conducted in the absence of any commercial or financial relationships that could be construed as a potential conflict of interest.

Received: 11 November 2008; paper pending published: 27 November 2008; accepted: 07 February 2009; published online: 18 February 2009.

Citation: Müller N, Schlee W, Hartmann T, Lorenz I and Weisz N (2009) Topdown modulation of the auditory steadystate response in a task-switch paradigm. Front. Hum. Neurosci. (2009) 3:1. doi: 10.3389/neuro.09.001.2009

Copyright (c) 2009 Müller, Schlee, Hartmann, Lorenz and Weisz. This is an open-access article subject to an exclusive license agreement between the authors and the Frontiers Research Foundation, which permits unrestricted use, distribution, and reproduction in any medium, provided the original authors and source are credited. 